\title{
Metaphysics of Controlled Evolution (Anthropic Principle, Evolutionary Epistemology and Ethics of Nano-Bio Technologies)
}

\author{
Valentin T. Cheshko \\ DS, prof., Academician - Foreign Member of Russian Acad. Natur. Sci.;, \\ V.N. Karazin Kharkiv National University, (Sq. Svobody, 4, Kharkiv, 61027, Ukraine)
}

\author{
Yulia V. Kosova \\ Lecturer, S.Kuznets Kharkiv National University of Economics, \\ (av. Nauki, 9A, Kharkiv, 61166, Ukraine ,)
}

Valery I. Glazko

DS, prof., acad. of RAS, The head of the Center for NanoBioTechnology, K.A. Timiryazev Russian State Agrarian University MSAU, Timiryazevskaya st., 49, Moscow, 127550, Russia.

\begin{abstract}
With the entry of anthropogenic civilization into the stage of the information society, the post-academic phase of the historical evolution of scientific rationality began, the attributes of which are the specific methodology of scientific knowledge, scientific ethos and ontology. Natural philosophy becomes the meta-theoretical core of modern theory of anthropogenesis and the conceptual basis of biotechnology. As the initial postulate of the metaphysical structure of modern technologies and the transdisciplinary theory of their implementation (social verification), the most promising is the anthropic principle of participation. It is used as a starting postulate, on the basis of which the concept of a stable adaptive human strategy is formulated. It is represented by three systems of generation, coding, implementation and replication of adaptively significant information: respectively, biological (genetic), sociocultural and technorationalistic adaptive modules. A necessary and sufficient condition for the genesis of this cycle was the formation and complication of the system jf information communication between individuals through the symbolic sign system not directly related to the genetic code. As a result, the spontaneous coevolutionary process of interaction between the "subject" (living organisms) and the "object" (material world), is the teleological trend of the movement towards the complete rationalization of the World as It Is, its merger with the World of Due. The trajectory of evolution is determined by three parameters that can potentially be estimated empirically: descriptive-spontaneous (evolutionary efficiency), value-teleological (evolutionary correctness) and integral (quality of life). This metasystem transition can be called adaptive inversion, which, therefore, is the most important attribute of anthropogenesis, in general, and the formation of civilization, in particular. Adaptive inversion was established, in our opinion, as a supporting structure of human evolutionary strategy, embodied and maintained as an irresistible cultural and psychological intention to transform reality. This intention, although in different ideological and cultural forms in different types of civilization, was most pronounced precisely in the transatlantic ("Western") version of the technogenic civilization. Renunciation of it and replacing of the desire to slow down the transformation of our habitat and to bring it into the framework of acceptable risk by something less radical seems incompatible with the "human nature." A human (Homo sapiens), as the carrier of the rational beginning of reality, acts as a condition for observance of the anthropic principle. The integrity of reality has a time-bound dimension and assumes the constant emergence, formation and overcoming of the subject-object (epistemological) antinomy and its ontological (essential - obliging) equivalent. Thus, the stratification of the global evolutionary process into selective and semantic (teleological)
\end{abstract}


coevolutionary and therefore ontologically inseparable components follows from the anthropic principle.

Keywords: anthropic principle, stable evolutionary strategy of Homo Sapience, evolutionary risk, technologies of controlled evolution, anthropogenesis, transdisciplinarity, post-academic science.

The evolution of science as a social institution and systemic socio-cultural adaptation that initiated the genesis of anthropogenic civilization passed several phases parallel to the evolution of the technogenic civilization itself. With the entry of civilization into the stage of the information society, a new phase of the historical evolution of scientific rationality, which is usually called "post-nonclassical" [Cheshko et al., 2015] or "post-academical» [Ziman, 2002: 83] science began. Its characteristic attributes are the specific methodology of scientific knowledge, scientific ethos and ontology.

The greatest importance in this list has the intertwining of three forms of discourse - the descriptive scientific, activity-based technological and public axiological ones. Their demarcation in modern human dimension theories is possible only situationally. This is the content of the most radical difference between "post-academic" scientific knowledge and its classical and non-classical variants.

This triad (science-technology-axiology), when it comes to a human or a self-organizing (evolving) system that includes human, forms "techno-knowledge" - an inseparable amalgam of logical arguments. This interpretation of the "techno-knowledge" category differs from the canonical one (as a matter of fact the technical sciences in the general classification of sciences). It is a matter of fundamental changes in the organization of the social institute of science and the scientific ethos that ensures its functioning [Ziman, 2004: 83], [Pruzhinin, 2013: 110], the structure of scientific knowledge [Cheshko et al., 2015] and the methodology of scientific research [Gibbons M. Et al., 1994: 90] Nowotny, 2003: 179] ..

The initial point here is the development of the NBIC technological complex. In its essence, it is a technology to control and improve the genetic, sociocultural and cognitive codes, allowing to control the course of biological and socio-cultural evolution of a person, i.e. The technology of controlled evolution applicable to Homo sapiens.

In our previous publications [Cheshko et al., 2014, 2015], we presented the arguments for a new concept of global bioethics as social practice and social institute of management of evolutionary risk of modern technologies of NBIC class (1) and, at the same time, as a carrying element of the disciplinary matrix of modern (post-academic) science with human dimension(2). Delimination of our interference in the natural process of evolution as applied to human is the prerogative of not natural science, but philosophy. Natural philosophy becomes the meta-theoretical core of modern theory of anthropogenesis and the conceptual basis of biotechnology. It means that in the era of biotechnology and genomics bioethics turns out a modern version of natural philosophy and, perhaps, metaphysics and rehabilitates natural philosophy as an explanatory model of the scientific theory of evolution. These changes are connected by the coevolutionary relations with changes in the world outlook basis and the mentality of modern civilization.

The purpose of this study was an attempt of transdisciplinary synthesis of anthropological, epistemological and ontological aspects of scientific and technological development. The main method of investigation was the creation of a deductive explanatory model of the significance of culture and scientific knowledge as factors of the macroevolutionary process. 
Ontological aspect of the anthropic principle in the transdisciplinary scientific-methodological paradigm

As the initial postulate of the metaphysical design of modern technologies and transdisciplinary theory of their implementation (social verification), the most promising is the anthropic principle, the corresponding to the problems of technologies of controlled evolution definition of which [Wheeler, 1977] says: «Observers are necessary to bring the Universe into being». Taking into account the realities created by nano-biotechnologies, the anthropic principle should be expressed as follows: only that Universe, in which there is an active agent endowed with the mind, acquires the status of Reality. From the subject and observer who cognizes the laws of nature, a person turns into the subject of activity, co-Participant and coCreator of reality.

Actually, the anthropic principle was explicitly formulated in 1973 by Brandon Carter [Carter, 1974: 290], although close concepts were voiced at least from the end of the 19th century. However, most of the canonical formulations, from our point of view, are notable for their anthropocentricity. But the principle of co-participation fixes the two aspects of reality simultaneously: first, the integrity of the Reality as a system in which the Subject and the object of reality are initially connected, and, secondly, the dual nature of this unity, simultaneously, communicative-informational and projectively-active. It is the latter circumstance (information-projective unity of reality) that can be united by a common attribute - coevolution. Reality exists as a coevolutionary relation of the Subject and the Object, and its content and becoming are simultaneously the result and cause of each of them. Homo sapiens simultaneously is determined and determines the status of the real world.

The coevolutionary interpretation of the anthropic principle becomes apparent when, together with the initial cyclone of the Subject-Object transformations, the second branch, the SubjectSubject (self) transformations, appears. Thus a kind of evolutionary hypocycle arises, where a purposeful transformation of the World is realized in parallel and interdependent with selfconstruction and self-manipulation of a rationally operating Subject. This double hypercycle is the phenomenological description of the current Reality as such. Equally, one can use the expression self-description, since the objective hypostasis of reality is the emergence of NBIC, which is also a consequence of the anthropic principle.

From this point, the global evolutionary process acquires a humanistic meaning, implying the Risk, generated by man, as an inevitable attribute of reality, monotonously approaching a unit in its magnitude and becoming evolutionary in its form. Science and technology are not only means of surviving of humanity and a source of power over the "raging" reality, but lead to the deviations that violate the anthropic principle - the correspondence of the parameters of the socio-natural habitat to the necessary and sufficient conditions for the existence of intelligent life in the Universe. This operator of obligation, responsibility of a person to reality logically follows from Wheeler's formulation and can not be traced explicitly in the canonical, "strong" and "weak" variants of B.Carter.

From the anthropic principle of participation the need for a transdisciplinary review of the three aspects of the problem of reality follows:

1. Ontological aspect is the reconstruction of the categorical apparatus by means of which the cognitive-projective image (Design) of the Reality is formed;

2. Epistemological aspect - creation of the conceptual field of the problem of communication Macro ("object") and Microcosm ("subject") as two attributes of reality, mutually conditioning and mutually determining each other; 
3. Anthropological aspect - disclosure of internal nature and temporal trends of the genesis of binary communication of Micro- and Macocosm. In the process of transition from ontology to anthropology, we move from the philosophical world-view level through evolutionary anthropology (meta-theoretical level) into the sphere of specifically-scientific theoretical studies.

The "three whales" of the new, transdisciplinary theory of scientific knowledge are ontological inactivism, evolutionary theory and the theory of co-evolution (the concept of a triple helix and the construction of a niche).

In their totality, they all proceed from the intention of overcoming Cartesian dualism (more precisely, Cartesian antinomy) of the object and subject of cognitive activity.

Enactivism claims [Varela et al., 1992 (2017): 185] that the process of cognition is not a process of forming an objectified reflection of material reality in the mind of human, but represents the creation of reality due to the coevolutionary interaction of the organism with their habitat. Accordingly, the object and the subject are an inseparable whole, the product of knowledge is not knowledge, but mutual adaptation of the world and the organism to each other.

In other words, in the course of cognition, a cycle of information and communication links is established, during the implementation of which a bilateral correspondence of the corporeal organization and environment is established.

In this interpretation, firstly, "knowledge" and "activity" correspond to two aspects of adaptational genesis. Secondly, the mind is somatically determined, its form follows from the body organization. And, thirdly, the differences between the physical (the "world as it is") and the virtual ("the world of due" and the "world of the possible") realities are imaginary, because they are simply a set of scenarios of global evolution.

Similarly, evolutionary epistemology came to the conclusion [Popper, 1972: 121 et al.], [Поппер, 2002], [Campbel, 1974: 141] Thomson, 1995: 165] on the identity of the process of adaptive evolution and cognition. The golmology between them follows from the same functional scheme:

$$
T T_{i-1} \rightarrow E E_{i} \rightarrow P P_{i} \rightarrow H H_{i} \rightarrow F F_{i} \rightarrow T T_{i} \rightarrow E E_{i+1} \rightarrow P P_{i+1,}-
$$

where $E E_{i}$ - data obtained empirically;

$P P_{\mathrm{i}}-$ problem situations, i.e. discrepancy between existing data and its theoretical explanation $\left(\mathrm{TT}_{\mathrm{i}-1)}\right.$;

$H_{i}$ - suggested explanatory models;

$F F_{i}$ - falsifiers, they deductively predict consequences which give possibility for empirical verification;

$T T_{i}$ - hypotheses that passed the falsification test and obtained the status of reliable theories; $E E_{i+1}$, and $P P_{i+1}$ - new data and new problem situations, discovered as a result of development of $\mathrm{TT}_{\mathrm{i}}$. 
The analogy between the process of cognition and adaptive evolution becomes apparent as a result of the comparison of (1) sets of data and gene collections, (2) a multitude of hypotheses and a multitude of mutations, (3) problematic situations and transformations of the ecological environment, (4) theories and biological populations /species, (5) procedures of falsification and natural selection. Thus, in this case, the identification of evolutionary adaptation and reliable knowledge seems logical. The growth of knowledge and the growth of adaptive complexity turn out to be entirely equivalent and stemming from the evolutionary strategy of Homo sapiens.

The contribution of "nominees" to the status of the paradigm of modern evolutionary theory in the analysis of the evolutionary aspects of social verification of the implementation of all varieties of NBIC-technologies has two conceptual roles: "constructing of (social) ecological niche" and "triple helix". The first one regards an evolutionary process with an emphasis on a static organization, the second on dynamic trends in the formation of the adaptive complexity of self-organizing systems.

According to the conceptual model of "niche construction" [Oding-Smee et al., 2003: 246], [Laland et al., 2016: 191-202], in the process of adaptation to the environmental conditions, living organisms change not only their own somatic and behavioral organization, but conditions of its own existence - spontaneously or (in the case of Homo sapiens) purposefully. Thus, in factl evolutionary process is considered within the framework of this explanatory model as co-evolution of living organisms and (socio) ecological niche. It is resulted in mutual adaptation, "fitting-in" of the niche conditions and the population of organisms that exploit it (niche).

The consequence of this is the famous law of Gause - one and the same ecological niche occupies by no more than one ecological species. In the alternative, we can observe either the extinction of other species or the division of the niche into several ones. A special feature of anthropogenesis is that the ascending branch of the coevolutionary cycle (organism $\rightarrow$ habitat) in its rationalistic form prevails over the specific gravity of the spontaneous-descending branch (habitat $\rightarrow$ organism). The general vector and specific trajectory of socio-cultural anthropogenesis in less degree is determined by environmental dynamics and becomes increasingly spontaneous (intentional). In our works this feature is called the evolutionary adaptive inversion (A. Zubov's expression). As a result, the ecological niche itself, as applied to a human, turns into a cultural-ecological niche and its boundaries are permanently expanding to the borders of the biosphere, after which a "spin-off" of microecological artificial ecosystems including a person begins.

The next concept explores the mechanisms of this co-evolutionary interaction - the so-called "triple helix" concept [Lewontin, 2002], [Leydesdorff, Franse, 2009: 109]. According to its logical organization, it is a post-Hegelian, post-Marxist interpretation of dialectics. As was shown at the beginning of the 20th century, binary Hegelian scheme can not explain the phenomenon of increasing adaptive system complexity with respect to co-evolutionary systems. "Struggle of opposites" (an example is the "predator-prey" system) ends with the formation of a stable binary opposition oscillating around an equilibrium position for indefinitely long period of time (the Volterra-Lotka model).

The phenomenon of the progressive increase in complexity (the Hegelian spiral) of selforganizing systems emerged due to the addition of the third element to the binary conjunction. With its emergence, the entire system acquires the ability to form a stable and irreversible 
evolutionary trend - either to complication of the organization, or to gradual erosion and destruction.

Before the emergence of human, systemic complexity was formed as a result of the functioning of the co-evolving triad genotype-phenotype-ecological system. The totality of the genome elements underwent epigenetic modifications in the process of realization of genetic information and formed the phenotype of organisms. In its turn, the survival of organisms was determined by their adaptation to the biotic conditions and abiotic parameters of the ecological niche and those transformations that vital activity of living beings brought to these parameters. All three elements of this triad were interdependent and connected by a complex network of direct and inverse influences, an essential role in which was played by informational communication between individuals (sociality).

\section{Three-modular organization of a stable adaptive strategy of human (anthropological aspect of the anthropic principle)}

Thus, the existence of social species has formed the prerequisites for a metasystem transition. The beginning of anthropogenesis became its realization.

A new coevolutionary triad has emerged that has become a substratum of a new cycle of producing systemic complexity. The foundation of this cycle is represented by a unique evolutionary phenomenon - the three-module stable evolutionary strategy of Homo sapiens (SESH), [Cheshko et al., 2015]. It is represented by three systems of generation, coding, implementation and replication of adaptively significant information: respectively biological (genetic), sociocultural and technorationalistic adaptive modules. A necessary and sufficient condition for the genesis of this cycle was the formation and complication of the information communication system between individuals through a symbolic sign system not directly related to the genetic code.

As a result, the spontaneous coevolutionary process of interaction between the "subject" (living organisms) and the "object" (material world) is the teleological trend of the movement towards the "Omega point" in the sense of Pierre Teilhard de Chardin [De Chardin, 2008 (1955): 66 et al.] i.e. to the complete rationalization of the World As It Is, its merging with the World of the Due. The subordination of the evolving Matter to the Logos took place; the transformation of life from the passive object of the evolutionary process into an active subject of constructing reality, and the reality itself transformed into the result of realization of an intellectual project. This metasystem transition, as already mentioned, can be called an adaptive inversion, which, therefore, is the most important attribute of anthropogenesis, in general, and the formation of civilization, in particular.

In our opinion, adaptive inversion was established, as the supporting structure of human evolutionary strategy, embodied and maintained as an irresistible cultural and psychological intention to transform reality. This intention, although in different ideological and cultural forms in different types of civilization, was most pronounced precisely in the transatlantic ("Western") version of the technogenic civilization. Turning it down and replacement of the desire to slow down and put transformation of our environment into the framework of acceptable risk with something less radical, appears to be incompatible with "human nature".

But the same consideration allows us to draw another conclusion: a stable adaptive strategy of Homo sapiens and adaptive inversion do not arise as a one-stage event (macromutation), but as a process and we can talk about both evolution of the evolutionary strategy itself and 
adaptive inversion. Indeed, the totality of the empirical data of evolutionary anthropology and the history of civilizations allows us to conclude that there have been several phases in the course of anthropogenesis, each of which is characterized by a specific form of organization of intermodular links within a stable evolutionary strategy. Together with these phase transitions in adaptive inversion, we can distinguish several recursive layers, supplementing the initial projective-activity intention (adaptive inversion in the original sense of this term).

Adaptive inversion radically changes criteria of selection of evolutionary innovations. The evolutionary success or failure of a sociocultural and then rationalistic innovation is determined by the dynamics of the transformation of saparate elements of the external environment into a life support resource for an individual and a social group. The adaptability of such an innovation stems from its ability to turn the components of the environment into a source of sustaining life and expanding the number of carriers of the same innovation. From the point of view of the evolutionary theory, there is a progressive multiplication of ecological niches available to Homo sapiens. At the same time, the biological nature of the carriers of adaptive innovation remains unchanged, at least in the final stages of anthropogenesis. In other words, the evolutionary divergence changes its nature - from genetic (biological speciation) becomes socioeconomic; ecology is replaced by the economy.

During the first phase of the evolution of $S E S H$, the fundamental trend in anthropogenesis is the dominance of the socio-cultural component of adaptational genesis over the biological one. The second phase is characterized by the splitting of the socio-cultural SESH module into two actually socio-cultural and techno-rational. Its beginning is the Neolithic revolution, the first technological system adaptation (the transition from hunting and gathering to farming and livestock farming, realized, however, purely in a sociocultural way.

Later, the intentional algorithm (typical for the sociocultural module) of solving cognitive tasks is replaced by a mechanical (physical) algorithm. An intensional algorithm is based on the decoding of the object's behavior in accordance with the analogy with his own behavior in the same specific situation: the interpretation of the object's behavior (a person, an animal, an artifact, anything), when he is perceived as if he was a rational agent, which in the case of "choice" of "action "is guided by" beliefs "and" desires " [Carruthers P. et al., 2005: 12-15, 153].

According to the mechanistic algorithm, the properties of the system are a superposition (linear totality) of its constituent elements, the forecast of the future is made as a result of unambiguous extrapolation of the change in the system and its elements with changing in external conditions. A distinctive feature of reality from the habitat is the binary opposition of the subject (the world of due) and the object (the world as it isThe traces of the bundle of intentional-constructive algorithms in the "evolutionary history" of anthropogenic civilization can be clearly traced in the philosophical and ideological tradition of deism of the 17th - 18th centuries. A balanced homeostatic structure of SESH is formed, where the system of value priorities (sociocultural module) plays the role of the balance-controller of the evolution vectors created by the biological and techno-rationalistic modules.

During the third and fourth phases of the evolution of SESH, several successive adaptive inversions occur during several decades of the last century. The term "recursion" means that each subsequent inversion does not negate the previous one, but occurs inside it.

So, an extraverted projective and activity behavioral intention (adaptive inversion 1), aimed at transforming of the reality in accordance with the interests and needs of human, recursively extends to the human himself - his genome, psyche and culture (adaptive inversion 2). The 
arena of evolutionary transformations is the technorational module. However, the actualization of the technological potential created by it is associated with the restructuring of the socio-cultural module and is accompanied by shocks of the ethico-legal and sociopolitical foundations of civilization ("eugenic movement in Western Europe and North America, racial hygiene" in Nazi Germany, "Michurin genetics and creative Darwinism" in aormer USSR).

As a compensatory reaction of the sociocultural module, an introvertive reorientation of the cognitive activity vector is observed from the scientific explanation of the surrounding world to scientific knowledge itself, which led to the stratification of the latter into a risky (classical) and warning science and the initiation of international socio-cultural mechanisms for monitoring the implementation of projective and activity behavioral intention (adaptive inversion 3). The manifestation of the development of the mentioned control mechanisms is the initiation and integration into the social life in general and its political sphere in particular bioethics and biopolitics, as social institutions that carry out such monitoring.

The presented concept at this point passes into the conceptual field of the proper theoretical science, as it allows not only social but also purely logical-empirical verification. The trajectory of evolution is determined by three parameters that can potentially be estimated empirically.

Evolutionary efficiency (inclusive adaptability) $\mathrm{E}$ is defined as the geometric mean value of the relative adaptivity $\mathrm{W}$ of all members of the evolving configuration, in our case - genome $(\mathrm{g})$, culture (c), and technology (st)

$$
E=\sqrt[3]{W_{g} W_{c} W_{s t}}
$$

However, if the adaptivity of the biological module is calculated with respect to individual genes and individuals in the population, then the adaptability of the socio-cultural module is applied to individuals and social groups, and the effectiveness of the rationalistic module is applied to social groups predominantly. Since this factor is not reflected in the formula of evolutionary efficiency, the quantity $\mathrm{E} \rightarrow 1$, but does not reach the latter.

In neo-Darwinism (the synthetic theory of evolution), biological (genetic) adaptivity turns out to be connected to the selection coefficient $s$ by the relation $W=1-s$. However, the threemodular structure of the adaptivity of SESH is determined not only by the selective factor, but also by the influence of the sociocultural and technological context, i.e. the adaptive significance of specific features and genes that are created by culture and technogenic modifications of living conditions. The content of these influences can be identified with the meaning that is given to the elements of adaptability by a system of value priorities (coevolutionary semantics). In their direction and magnitude, they, as a rule, do not succumb to selective pressure. As a result, the total value of adaptivity is defined as the resultant of selective $\left(\mathrm{s}_{1}\right)$ and semantic $\left(\mathrm{s}_{2}\right)$ factors: $W=1-s_{1}+s_{2}$. In other words, the virtual values of the components of $E$-parameter should exceed unity, which contradicts the definition of $W$. The same arguments can be applied to the implementation of technological innovations: the "success" or "failure" of integrating technological innovations is determined both by their own effectiveness and the relationship with the system of values norms and priorities of a sociocultural type.

Many inherited pathologies, like diabetes, phenylketonuria, congenital dislocation of the hip, in traditional society had an adaptability equal to zero. In modern civilization, they are characterized by survival and reproductive potential, practically indistinguishable from the norm $\left(E=1, s_{2} \rightarrow+1\right)$. This corresponds to the values of the sociocultural and 
technorationalistic components above 1 . Then there is the possibility of eliminating those elements, the biological component of efficiency, the negative emergence of which are observed outside the reproductive age (Alzheimer's disease, Huntington's chorea, the diabetes). This phase of development of the technorationalist module is equal to a drop in biological adaptability on these grounds to zero $\left(s_{2}-s_{1} \rightarrow 1\right) /$. And, finally, the moment comes when the biological module undergoes genetic manipulation and some of its elements are eliminated (Human Genome Enhancement).

In all these cases, the value of $\mathrm{E}$ changes abruptly, and the selective component of adaptational genesis does not have time to "notice" it. Such quantum transitions are equal to changes in the communicative semantic code of intermodular communication (changes in the adaptive significance of the elements of one module under the influence of other modules). The above arguments ultimately forced us to abandon the use of the term "inclusive (integral) adaptivity" that suggested itself in favor of "evolutionary effectiveness".

As a result, the evolutionary process undergoes a dichotomy and is divided into two evolutionary mechanisms - classical Darwinian selection and evolutionary semantics provided by the sociocultural module. The very existence of such a phenomenon (co-evolutionary semantics [Cheshko et al., 2015: 256]) indicates the existence of the second - the subjectivevalue parameter of the evolution of reality.

Evolutionary correctness of $K$ is determined by the discrepancy $(L)$ between the maximally effective and optimal scenarios of evolution in accordance with the set of value priorities that dominates in society

$$
K=\left(1-\frac{d L}{d t}\right)
$$

Empirically, this value can be defined as the sum of the parameters $\left(\mathrm{f}_{\mathrm{i}}\right)$, on the basis of which (self) identification of a person occurs (referring an individual to humanity or giving up (dehuman) such identification). For the purpose of transfer into a dimensionless form, it correlates with the total number of factors of humanization/dehumanization $-N$ :

$$
V=\sum\left(f_{\text {human }}-f_{\text {dehuman }}\right) /_{N}
$$

These transformations allow us to talk about the semantic nature of evolutionary correctness, as we suggested above. (The concept of co-evolutionary semantics was suggested by B. Crespi several years ago [Crespi, 2010.]) There is a necessity to find an integrative criterion that allows to take into account the evolutionary trajectory of our biological species and the evolution of eco-social systems (with human dimention) of different levels - up to the biosphere - results of the nonlinear interdependence of both indicators.

Paradoxically, we find this criterion in empirical sociology.It may seem strange only at first glance: sociological research, when appropriate, integrates the influence of objective (interests) and subjective (values) factors on the behavior of the totality of individuals members of society.

"Quality of life" QoL - a measure of individual and group contentment in socio-ecological conditions of existence; the product of values of evolutionary efficiency (inclusive adaptability) and evolutionary correctness

$$
Q o L=E K
$$


David Wilson, one of the authors of the theory of multilevel selection, considers this category to be logically related to the explanatory model of niche construction [Wilson, 2016: 333]. We can agree with this thesis, however, with the clarification that the quality of life in this case can be defined as a measure of the synergy of both factors of evolution. Taking into account their vector nature (resulting from their mutual correlation), the dynamics of the change in the quality of life in time is described by the two-sided functional dependence of evolutionary efficiency and evolutionary correctness

$$
\frac{d Q o L}{d t}=\frac{d[\vec{E}(K) \vec{K}(E)]}{d t}
$$

As we can see, from the successive application of the anthropological principle in its Wheeler's interpretation it follows that a change in the "human nature" in this case is equival to a transition to an alternative reality. We have to agree with V. V. Kazyutinskim [Cheshko et al., 2015] about the revival of the selective subjectivism of Arthur Eddington, as a consequence of the anthropic principle of participation. However, a tandem biotechnology-bioethics contributes to this thesis a substantial and radical specification: selection of objective schemes of the world perception preexisting in our mind during the Human enhancement process (selfconstruction of human) finishes in fixation of the ideal scheme of a new "physical" reality, that came through the filter of selection. It means that biopolitics and bioethics as elements of the socio-cultural adaptive module of SESH, and biotechnology and humanitarian technologies, as components of the technorational adaptive module, become the most important factors in the evolutionary process. It is interesting, a conceptual apparatus for taking into account the objective (evolutionary efficiency) and subjective (evolutionary correctness) parameters of the evolutionary process (analogues of which in the social and political theory are the concepts of "interests" and "values") has already been created in the sociological paradigm.

In social anthropology, it is agreed to mark out two alternative concepts. In accordance with the naturalistic concept, the activity intention is defined by the multidimensional topos of interests as an individualized subjective mental reflection of the objective parameters of the most favorable objective environmental reality. In terms of ontology - the world as it is and the world of due are connected by the network of cause-effect relationships, although not necessarily unambiguous.

In the transcendental concept, that origins in the works of Immanuel Kant and David Hume, the projective activity system of intentions is determined by objective values, i.e. the divergence between reality as it is, and the world as it should be, and the world can not be deduced logically from existence. Thus, values are conditioned by culture as a counterweight and antithesis to the biological and economic factors for life.

In the coevolutionary interpretation, the concepts of "interests" (needs) and "values" reflect alternative aspects of the real evolution of SESH. Then, in relation to culture, interests act as external, conditioned by genetic and rationalistic components (evolutionary effectiveness), and values - as internal, culturologically determined factors of the definition of a set of evolutionarily correct scenarios.

The mechanism through which biopolitics and bioethics are re-transmitted into the factors of the evolutionary process can be called ontological conversion: biopolitical intentions and postulates and bioethical norms and imperatives acquire ontological significance, and become elements of the image [Dasign] of a desired, acceptable or unacceptable future, the ontological status of practical and theoretical politics is mediated by ethics [Pellizzoni: 2016: 5]. As a 
result, a new coevolutionary cycle appears in the socio-cultural module, producing a systemic complexity of the current of global evolution with human participation.

A similar idea of socio-technical images ( «Sociotechnical Imaginaries») as a factor of biosocial evolution of human independently and simultaneously was formulated by Sheila Jasanoff [Jasanoff, 2015: 1-34]. With reference to techno rationalistic component of SASH ideological precursor of both hypotheses is introduced by Michel Foucault concept of epistemes [Foucault, 2002 (1969): 197] as the set of hidden historically caused cultural and cognitive a priori preconditions determining the shape of mental processes, due to which the content and borders of scientific knowledge are formed. The integration of the idea of the episteme into the general construction of the evolutionary epistemology of Karl Popper leads to our interpretation.

At the same time, biopolitics provides a progressive (projectively-activity) side of evolutionary correctness of global evolution; i.e. the best possible correspondence of evolutionary efficiency $-\lim (E-K)=0$. Bioethics realizes the appraisal-stabilization function, i.e. provides the maximum possible approximation of evolutionary correctness to the ideal $-\lim K=1$. Accordingly, the indicator of acceptable values of evolutionary civilizational and technological risk $(\mathrm{R})$ is the maximum possible quantities of quality of $\operatorname{life}-\lim R=(1-Q o L)=(1-E K)=$ 0

\section{Binary opposition biotechnology-bioethics in the paradigm structure of the scientific theory with human dimension (epistemological aspect of the anthropological principle)} According to our conception, bioethics and biotechnology together with the belt of theoretical (biotechnology) and applied (bioethics) developments that surrounds them (bioethics and biotechnology) is a "double reflection" of the paradigmatic structure of each other, because in paradigms of both areas of knowledge the elements of the descriptive-scientific and valuepublic discourse are combined (a phenomenon that in the framework of classical positivist epistemology seemed to be an obvious "heresy").

Bioethics as a phenomenon of intellectual culture can be presented as a natural philosophical methodological core of modern post-academic science (with human dimention), in which the principle of ethical neutrality of the scientific theory is inapplicable, and elements of public axiological and scientific descriptive discourses are integrated into a single logical construction. As a consequence, hermeneutics precedes epistemology not only methodologically, but also semantically, and natural philosophy restores the status of a major element of the theory of evolution, and, moreover, in an explicit form. The theoretical basis of biotechnology and other convergent technologies that open up the possibility of managing/manipulating the evolution of self-organizing systems that include the mind-bearer, in our opinion, is the concept of the stable evolutionary strategy of Homo sapiens.

In the case of antiparallel orientation of vectors of evolutionary efficiency and evolutionary correctness, the characteristic value of risk crosses the boundaries of the "physical" meaning very quickly. The achievement of this point means the irreversible destruction of the system of value priorities, the central core of which is the concepts of humanity and human nature.

This interpretation demonstrates the potential and actual risk of both genetic and sociological reductionism in the bioplitics of modern society. The danger of genetic reductionism, which relies on the possibility of solving all evolutionary conflicts arising as a result of the dysfunctionality of the human genome and socioecological niche created and occupied by the 
Homo sapiens, is obviouse by purely genetic engineering method. It meets the most positive response among the specialists in the Humanities and in the mass consciousness that forms the electorate of modern Western democracies.

The danger of sociological (cultural) reductionism, in our opinion, meets support in the scientific community primarily of naturalists, although its consequences can be as inhumane as the uncontrolled (bio) technological imperative.

As Wilson D.S. [Wilson D.S., 2016: 335] ironically mentioned, happiness and contentment, like the rest of human emotions, are merely adaptive mechanisms that provide short-term reproductive success. In the three-module SESH, this emotion "happiness" and its corresponding psychophysiological state of euphoria serves as a means of psychological motivation [Grinde B., 2012: 20], which is a part of the complex of ensuring the evolutionary success of human beings - active and passive self-preservation, life support and reproduction.

\section{CONCLUSION}

\section{Let's translate all of the above into the language of ontology.}

The participatory anthropic principle of Wheeler, because of its cosmological-existential content, is the central initial principle of any ontology of human nature in the philosophical meaning of this word (the nature of human as the essence of the carrier of the rational principle in the Universe). The second metaphysical principle throws a bridge to the anthropology of the genes and other technologies of controlled evolution. It affirms the threemodule organization of the evolutionary strategy of Homo sapiens as the carrier of Reason as the cause and mechanism for the realization of the anthropic principle of participation. As a result of discrepancies in the speed of biological, sociocultural and techno-rationalist evolution and the presence of coevolutionary relations between them, the evolution process splits into an objectively spontaneous and subjective-teleological components. For the same reasons, periods of relatively quiet development are replaced by evolutionary crises in due to the accumulation of conflicts between sets of elements of the biological, sociocultural and technorationalist modules. The solution of these conflicts is achieved either through the fixation of biological mutations, or by reformatting the sociocultural niche, or through technological interventions in the psychosomatic constitution and cultural stereotypes of human.

Using both of these principles as premises of deductive conclusion, we get the thesis about the global evolution of the systems with human dimension as a sequence of recursive adaptive inversions, during which the object and subject of reality transformations change their places. In this case, each subsequent projectively-activity inversion does not cancel the previous one, but embed into it. It imparts nonlinear and open characterthe to the evolutionary trajectory..

From this metaphysical triad, as you can see, it is possible to construct an ontological conceptual-terminological framework at the output of which we obtain logically consistent theories of constructing a socioecological niche, multi-level selection and the concept of evolutionary technogenic risk, evolutionary efficiency, evolutionary correctness and quality of life. With the help of these logical constructs, it becomes possible to create verifiable explanatory models of socio-cultural anthropogenesis and, in particular, a description of the evolutionary consequences of scientific and technological development.

The essence of the unique phenomenon of stable evolutionary strategy of our biological species is evidently most adequately and capaciously expressed by Elena Knyazeva [Knyazeva, 2014: 16]: "The constructing person and the world he constructs constitute a procedural unity". The 
key in this thesis is the "procedural" attribute. The integrity of reality has a temporal dimension and implies the constant emergence, formation and overcoming of the subjectobject (epistemological) antinomy and its ontological (essential - necessity) equivalent. The resultant of both oppositions at each instant of time will determine the instantaneous value of the vector of evolution of reality, however the state of reality itself is discrete and corresponds to the dominance of

either quanta of essence and objectivity (the transformation of knowledge and the ideal "world of due" in accordance with the state of the Object)

or the quanta of necessity and subjectivity (transformation of the Object in accordance with the state of Knowledge about the "World as It Is" and the predispositions of "World of Due").

During the first and second phases of the evolution of SESH, as a result of adaptive inversion, the "habitat" splits into the "world of the objective-existential (the world as it is)" and "the world is projective-ideal (the world of due") and thereby it turnes into "reality." This splitting was associated with the initiation of anthropogenesis and the emergence of consciousness.

Two outlines of cyclic causal relationships were formed. The first is the binary opposition - the body-an ecological niche. The splitting of the world in the second outline (the human intellect) permanently increases before the emergence of the technogenic civilization and the Cartesian antagonistic dualism "The Object of knowledge is the Subject of knowledge." After this, the stage of "removal", overcoming of this contradiction, restoring the integrity of reality due to scientific and technological "progress" begins. Actually, this is the globally evolutionary significance of the latter. With the introduction of technogenic civilization into the stage of information society, characterized by the development of technological schemes for controlling genetic, cognitive and sociocultural codes, the fourth phase of evolution of the stable evolutionary strategy of mankind begins. It can be defined as an approximation of the era of post-humanism, i.e. Another global evolutionary singularity. The evolutionary singularity is the bifurcation point, which denotes a radical change in the mechanisms of evolution. Thus, the whole history of the biological species of Homo sapiens fits into the intersyngulation period: from the stratification of the world into the subject and object of the cognitivetransformational activity of the "habitat", to overcome of this contradiction as a result of the self-construction of the intelligent life (Human Enhancement).

Let us return to the epistemological aspect of our study. The stage of the pre-industrial society (the Moderna era) corresponds to the disposition 0, Industrial society to the quantum transition $0 \rightarrow 1$, the modern status (postmodern) of the evolution of civilization corresponds to the dispositions 1 . The transition from classical scientific rationality to nonclassical and, after it to post-academic science, occurs conjoined.

Unlike the classical (disciplinary) matrix, the paradigm of postacademic scientific theory is a binary bundle of the descriptive and axiological core, as a result of the intersection of epistemological (scientific) and axiological (public) discourses.

The three-module model of the stable evolutionary strategy of Homo sapiens acts as a descriptive abstract-theoretical core. A distinctive feature of this model is the thesis of the rationalization of the global evolutionary process and the generation of an ever-growing evolutionary risk as the main attributes of SASH. 
Bioethics is a metatheory, which, we hope, is able to serve as a stabilizer of the system of attributes-identifiers of a human identity, as well as a system of cultural-mental predispositions that are formed on their basis. Such a system ensures the maintenance of the current version of the evolutionary semantics of the NBIC-technological complex within the "universal values" that ensure the preservation of mankind in the process of permanent development of technologies directed to the subject of the evolutionary process.

Thus, bioethics serves as the axiological core of the transdisciplinary matrix of synthetic biology and biotechnology.

The triad of the evolutionary strategy splits into two cycles, moving relative to each other:

The cycle of mutual transformations of physical reality (dichotomy of object - subject) as an object of ontology and

The cycle of transformation of the ideal reality (the world as it is - the world of due) as a subject of epistemology.

But each of these cycles not only assumes, but also determines the instant vector of the evolution of its partner. Contingency assumes that existence is only a means of achieving the stated goal, whereas the objectivity of knowledge requires descriptive conditioning of the stated goals by the initial conditions of existence. Both cycles are parsed and fused into a node at the points where the fragments of material reality finds their semantic meaning. Within the framework of the conceptual field of the SESH concept, these points are equivalent to acquiring an adaptive value that is sufficient for the implementation of the metasystem transition. After this, reality acquires the property of discreteness of the quantum leap from one of its hypostasis to another due to both the human activity and the spontaneous (not anticipated in advance) result of development. The omega point moves as it is approached in the $\mathrm{N}$ dimensional space by goals and the ways of their achievement. It is impossible to return and check the alternative scenario after passing the point of no return. Evolution leads to equally unpredictable results, as well as the realization of the Intelligent Design [Cheshko et al., 2015].

The human (Homo sapiens), as the carrier of the rational beginning of reality, acts as a condition for compliance of the anthropic principle. The actualization of the world constants necessary for the actualization of the being of a rational subject is procedurally determined by the reality project created by him. The correspondence between the objective and the subjective components of reality as a result of the implementation of this project is in any case not violated (more precisely, it disappears only in the case of self-distruction of bearer of Ratio). Simply a different reality arises, it has a different subjective and irrational manifestation (within the framework of Cartesian antinomy - another object and subject) and a different system of coevolutionary connotations between them.

\section{References}

Campbell D. T. Evolutionary Epistemology // The philosophy of Karl R. Popper. Ed. by P. A. Schilpp. LaSalle: Open Court, 1974. P. 412-463

Campbell D. T. Unjustified variation and retention in scientific discovery // Studies in the philosophy of biology: Reduction and related problems. Ed.by Fr. J. Ayala,Th. Dobzhansky. London; Bastingstoke: Macmillan, 1974. P. 141-161

Carruthers P., Laurence S., Stich S. (ed.). The Innate Mind: Vol. 1.Structure and Contents. - Oxford: University Press, 2005.449 p.

Carter B. Large number coincidences and the anthropic principle in cosmology //Symposium-International Astronomical Union. - Cambridge: University Press, 1974. - Vol.. 63. - P. 291-298. 
Cheshko V.T., Glazko V.I., Kosovsky G.Yu., Peredyadenko A.S.// Stable adaptive strategy of homo sapiens and evolutionary risk of high tech. Transdiciplinary essay. / Moscow, Novie pechatnie tekhnologii, 2015, 252 p.

Cheshko V.T., Ivanitskaya L. V., Kosova Y. V. Configuration of Stable Evolutionary Strategy of Homo Sapiens and Evolutionary Risks of Technological Civilization (the Conceptual Model Essay) // Biogeosyst. Tech., 2014, Vol.1, № 1. P. 58-69.

Cheshko V.T., Ivanitskaya L. V., Kosova Y. V. Evolutionary Semantics of Anthropogenesis and Bioethics of NbicTechnologies // Biogeosystem Technique, 2015, Vol.(5), Is. 3, pp. 256-266

Crespi B. J. The origins and evolution of genetic disease risk in modern humans // Ann. N.Y. Acad. Sci. 2010. Vol. 1206. P.80-109

De Chardin P. T. The phenomenon of man. - N.Y.; L.: Harper, 2008. 162 p..

Foucault M. The order of things: An archaeology of the human sciences. - N.Y.; L.: Psychology Press, 2002.422 p.

Gibbons M. et al. The new production of knowledge: The dynamics of science and research in contemporary societies. - Los Angeles; L.; New Delhi; Singapore; Washington DC: Sage, 1994. - 192 p.

Jasanoff S., Kim S. H. (ed.). Dreamscapes of modernity: Sociotechnical imaginaries and the fabrication of power.Chicago: University of Chicago Press, 2015. 363 p.

Laland K., Matthews B., Feldman M. W. An introduction to niche construction theory //Evolutionary Ecology. 2016. - Vol. 30. - No. 2. - P. 191-202.

Lewontin R. C. The triple helix: Gene, organism, and environment. - Harvard: University Press, 2002.144 p.

Leydesdorff, L., Franse, S. The Communication of Meaning in Social Systems // Systems Research and Behavioral Science. 2009. 26, No 1. P. 109-117.

Nowotny H. "Mode 2" Revisited: The New Production of Knowledge. Nine years ago, six authors published The New Production of Knowledge : The Dynamics of Science and Research in Contemporary Societies // Minerva. 2003. - Vol. 41. - P. 179-194

Odling-Smee F. J., Laland K. N., Feldman M. W. Niche construction: the neglected process in evolution. - Princeton University Press, 2003. - 246 p..

Pellizzoni L. Ontological politics in a disposable world: the new mastery of nature. - N.Y.; L.: Routledge, 2016.268 p.

Popper K. R. Objective Knowledge: An evolutionary approach. Oxford: Univ.Press, 1972. 402 p.

Thomson P. Evolutionary Epistemology and Scientific Realism // Joumal of Social and Evolurionary Systems 1995 Vol. 18, No 2. P. 165-191.

Varela F. J., Thompson E., Rosch E. The embodied mind: Cognitive science and human experience. - Cambridge; L.; MIT press, 2017. $392 \mathrm{p}$

Wheeler J. A. Genesis and observership // Foundational Problems in the Special Sciences - Dordrecht; Boston: Reidel Publ.Co, 1977. - P. 1-33.

Wilson D. S. Quality of Life from an Evolutionary Perspective //Applied Research in Quality of Life. - 2016. - Vol. 11. - No 2. - P. 331-342

Ziman J. Real Science. What it is, and what it means. Cambridge, UK: University Press, 2002. - 412 p. 